\title{
Nonorientable Genus of Nearly Complete Bipartite Graphs*
}

\author{
Bojan Mohar
}

Department of Mathematics, University E. K. of Ljubljana, Jadranska 19, 61111 Ljubljana, Yugoslavia

\begin{abstract}
Let $G(m, n, k), m, n \geq 3, k \leq \min (m, n)$, be the graph obtained from the complete bipartite graph $K_{m, n}$ by deleting an arbitrary set of $k$ independent edges, and let

$$
f(m, n, k)=[(m-2)(n-2)-k] / 2
$$

It is shown that the nonorientable genus $\tilde{\gamma}(G(m, n, k))$ of the graph $G(m, n, k)$ is equal to the upper integer part of $f(m, n, k)$, except in trivial cases where $f(m, n, k) \leq$ 0 and possibly in some extreme cases, the graphs $G(k, k, k)$ and $G(k+1, k, k)$. These cases are also discussed, obtaining some positive and some negative results. In particular, it is shown that $G(5,4,4)$ and $G(5,5,5)$ have no nonorientable quadrilateral embedding.
\end{abstract}

\section{Introduction}

Let $G(m, n, k)$ denote the graph obtained from the complete bipartite graph $K_{m, n}$ by deleting $k$ independent edges, where, of course, $k \leq \min (m, n)$. Then $G(m, n, 0)$ is $K_{m, n}$ and $G(n, n, n)$ is " $K_{n, n}$ minus a 1 -factor." These graphs are said to be nearly complete bipartite graphs. We are concerned with the nonorientable genus of the graphs $G(m, n, k)$ and, to avoid trivialities, we always assume both $m, n \geq 3$. Let

$$
f(m, n, k)=((m-2)(n-2)-k) / 2 .
$$

An easy application of Euler's formula implies the lower bound on the nonorientable genus:

$$
\tilde{\gamma}[G(m, n, k)] \geq\lceil f(m, n, k)\rceil .
$$

\footnotetext{
* This work was supported in part by the Research Council of Slovenia, Yugoslavia.
} 
It will be shown that equality holds in (1) in almost all cases, except in the trivial cases where $f(m, n, k) \leq 0$ (in this case the nonorientable genus is equal to 1 ) and possibly in some exceptional cases, the graphs $G(k+1, k, k)$ and $G(k, k, k)$. Equality in (1) will be proved also for graphs $G(4 k, 4 k, 4 k)$ (see Section 3, Theorem 2 and Proposition 1), $k>1$. For $G(5,4,4)$ and $G(5,5,5)$ it is shown in Section 5 that in (1) strict inequality holds, i.e.,

$$
\tilde{\gamma}[G(5,4,4)]=2 \quad \text { and } \quad \tilde{\gamma}[G(5,5,5)]=3 \text {. }
$$

Besides these interesting nonexistence results, the main result of this paper is:

Theorem 1. Let $m, n \geq 3, k \leq \min (m, n)$ and suppose that the triple $(m, n, k)$ does not fall into any of the following cases: $(k+1, k, k),(k, k+1, k)$ with $k$ even, or $(k, k, k)$ with $k$ arbitrary. Then

$$
\tilde{\gamma}[G(m, n, k)]=\max \{\lceil f(m, n, k)\rceil, 1\} .
$$

Only some special cases of Theorem 1 were known before. Ringel [6] determined $\tilde{\gamma}(G(m, n, 0))=\tilde{\gamma}\left(K_{m, n}\right)$, and Bouchet [2] constructed nonorientable genus embeddings of $K_{m, n}$ by using surgery methods very similar to ours. Our paper continues the work of Mohar et al. [5] where the (orientable) genus of all nearly complete bipartite graphs $G(m, n, k)$ is determined. On the other hand, genus embeddings of some more "general" bipartite graphs with many edges can also be obtained using similar methods as in this paper.

For additional background on genus and embeddings of graphs, we refer to $[1],[7]$, and [8].

\section{Surgical Techniques}

We consider only connected spanning subgraphs of simple complete bipartite graphs. We regard the first (or left) half of the bipartition as black vertices, and the second (or right) half as white vertices. A vertex is saturated if it is adjacent to all vertices of the opposite color; otherwise it is unsaturated. For example, $G(6,4,4)$ has six black vertices (of which two are saturated) and four unsaturated white vertices. All embeddings of graphs will be cellular embeddings in closed surfaces. Usually the embeddings will be quadrilateral, that is every face (or "2-cell") will be a 4-gon. The consecutive neighbors of a given vertex relative to an embedding will be the (cyclic) list of all neighbors as they are encountered when one traverses clockwise (or counterclockwise) a small circle, around the given vertex, on the surface. For quadrilateral embeddings, two neighbors of a vertex $v$ will be consecutive exactly when they lie in a common quadrilateral containing $v$.

If we switch the two colors, we obtain an isomorphism of $G(m, n, k)$ with $G(n, m, k)$. Therefore we need only prove any results for one case; the other case will then follow by duality. 

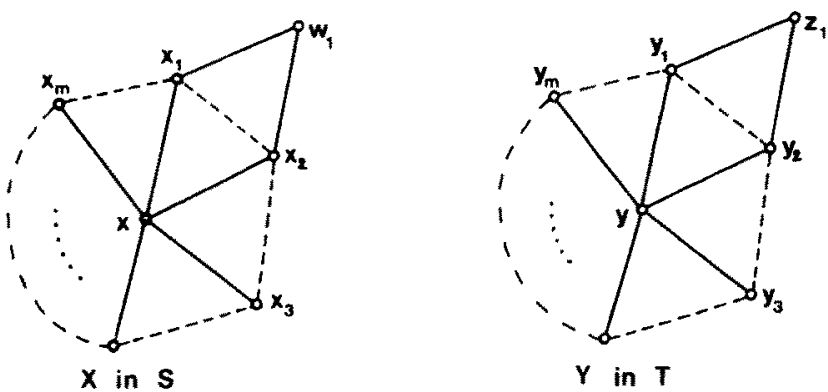

Fig. 1

We shall use two surgical lemmas for constructing quadrilateral embeddings. The first is a special application of a surgery method used previously by Bouchet [2], by Magajna et al. [4], and described in the same way as here by Mohar et al. [5]. Although definable in greater generality, the method will be stated as it applies to quadrilateral embeddings only, since we need only this special case.

Suppose that $X=G\left(m, n_{1}, k_{1}\right)$ and $Y=G\left(m, n_{2}, k_{2}\right)$ have quadrilateral embeddings in surfaces $S, T$ and have saturated white vertices $x$ of $X$ and $y$ of $Y$ (so, in particular, we must have $k_{1}<n_{1}$ and $k_{2}<n_{2}$ ) with the properties that their consecutive neighbors may be labeled as $x_{1}, \ldots, x_{m}$ and $y_{1}, \ldots, y_{m}$ (respectively) such that for $i=1, \ldots, m$, it is always the case that at least one of $x_{i}, y_{i}$ is saturated. If we introduce auxiliary edges $x_{i} x_{i+1}$ and $y_{i} y_{r+1}(i=1, \ldots, m$ and subscripts reduced $\bmod m$ ), we triangulate all the quadrilaterals containing $x$ and $y$ (see Fig. 1). If we then excise the disks containing $x$ and $y$ and bounded by the cycles $x_{1} x_{2} \cdots x_{m} x_{1}$ and $y_{1} y_{2} \cdots y_{m} y_{1}$ (respectively), and then sew the surfaces together along these boundaries so as to identify $x_{i}$ with $y_{i}$ and $x_{i} x_{i+1}$ with $y_{i} y_{i+1}(i=1, \ldots, m$ and subscripts reduced $\bmod m)$, and finally suppress the auxiliary edges, then we obtain a copy of the graph $X \#, Y=$ $G\left(m, n_{1}+n_{2}-2, k_{1}+k_{2}\right)$ quadrilaterally embedded in the connected sum $S \# T$ of the surfaces $S, T$. We refer to [5] for a detailed proof of this. The embedding of $X \#_{1} Y$ described above is orientable if and only if both $S$ and $T$ are orientable.

To distinguish various possibilities of orientability of embeddings we introduce the following notation:

$O(m, n, k)$ is the graph $G(m, n, k)$ quadrilaterally embedded into an orientable surface,

$N(m, n, k)$ is $G(m, n, k)$ quadrilaterally embedded into a nonorientable surface, and

$S(m, n, k)$ stands either for $O(m, n, k)$ or for $N(m, n, k)$.

Depending on the orientability of embeddings of $X$ and $Y$ in the above construction, we write

$$
\begin{aligned}
& O\left(m, n_{1}+n_{2}-2, k_{1}+k_{2}\right)=O\left(m, n_{1}, k_{1}\right) \#_{1} O\left(m, n_{2}, k_{2}\right), \\
& N\left(m, n_{1}+n_{2}-2, k_{1}+k_{2}\right)=N\left(m, n_{1}, k_{1}\right) \#_{1} S\left(m, n_{2}, k_{2}\right), \\
& N\left(m, n_{1}+n_{2}-2, k_{1}+k_{2}\right)=S\left(m, n_{1}, k_{1}\right) \#_{1} N\left(m, n_{2}, k_{2}\right), \\
& N\left(m, n_{1}+n_{2}-2, k_{1}+k_{2}\right)=N\left(m, n_{1}, k_{1}\right) \#_{1} O\left(m, n_{2}, k_{2}\right),
\end{aligned}
$$


etc., when two quadrilaterally embedded graphs can be combined to produce another as described above. The subscript 1 on $\#_{1}$ is to distinguish it from the symmetrical construction

$$
S\left(m_{1}+m_{2}-2, n, k_{1}+k_{2}\right)=S\left(m_{1}, n, k_{1}\right) \#_{2} S\left(m_{2}, n, k_{2}\right)
$$

which arises from the two quadrilaterally embedded graphs $G\left(m_{1}, n, k_{1}\right)$ and $G\left(m_{2}, n, k_{2}\right)$ upon excising two saturated black vertices $x, y$ whose consecutive neighbors can be labeled appropriately. Formally, we have:

\section{Lemma 1.}

(1) If $k_{i}<n_{i}(i=1,2)$ and $k_{1} k_{2}<m$ and both $G\left(m, n_{1}, k_{1}\right)$ and $G\left(m, n_{2}, k_{2}\right)$ have quadrilateral embeddings, then $G=G\left(m, n_{1}+n_{2}-2, k_{1}+k_{2}\right)$ has a quadrilateral embedding.

(2) If $k_{i}<m_{i}(i=1,2)$ and $k_{1} k_{2}<n$ and both $G\left(m_{1}, n, k_{1}\right)$ and $G\left(m_{2}, n, k_{2}\right)$ have quadrilateral embeddings, then $G=G\left(m_{1}+m_{2}-2, n, k_{1}+k_{2}\right)$ has a quadrilateral embedding.

In each case, the embedding of $G$ is nonorientable if and only if at least one of the initial embeddings is nonorientable.

The full proof of Lemma 1 can be found in [5]. We note only that the inequality $k_{1} k_{2}<m$ (resp. $k_{1} k_{2}<n$ ) is used to show that consecutive neighbors of the two chosen saturated white (resp. black) vertices can be labeled so as to satisfy the constraint that for each $i$, either $x_{i}$ or $y_{i}$ is saturated.

To produce embeddings, we shall also need another, more involved surgery lemma.

Lemma 2. Let $x, y \in\{1,2\}$. Suppose that there exist quadrilateral embeddings of graphs $G\left(m_{1}, n_{1}, k_{1}\right), G\left(m_{2}, n_{2}, k_{2}\right), G\left(m_{1}, n_{2}, x\right)$, and $G\left(m_{2}, n_{1}, y\right)$, where $k_{1} \geq 1$, $k_{2} \geq 1, m_{1} \geq k_{1}+x-1, n_{1} \geq k_{1}+y-1, m_{2} \geq k_{2}+y-1$, and $n_{2} \geq k_{2}+x-1$. Then there is a nonorientable quadrilateral embedding of the graph

$$
G\left(m_{1}+m_{2}-2, n_{1}+n_{2}-2, k_{1}+k_{2}+x+y-4\right) .
$$

Proof. Take the four graphs of the lemma embedded quadrilaterally into corresponding surfaces. In each of them choose a white and a black vertex which are not adjacent. Note that this is always possible since in none of the cases we do have a complete bipartite graph. Around each of the chosen vertices excise the disk just in the same way as we did in the construction of Lemma 1 . Since we took pairs of nonadjacent vertices of different colors, in each case a surface with two (disjoint) holes is obtained. The graphs which are so far embedded into these surfaces with boundary, say $S_{1}, S_{2}, S_{3}, S_{4}$, are $G\left(m_{1}-1, n_{1}-1, k_{1}-1\right)$, $G\left(m_{2}-1, n_{2}-1, k_{2}-1\right), G\left(m_{1}-1, n_{2}-1, x-1\right)$, and $G\left(m_{2}-1, n_{1}-1, y-1\right)$, respectively. Note that all the vertices (but no edge) lie on the boundaries. 


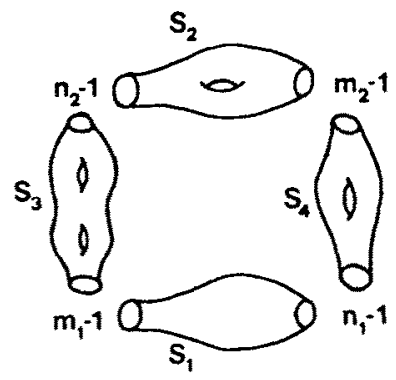

Fig. 2

Next orient the eight boundary cycles (two on each surface) as follows. If all surfaces $S_{i}$ are orientable, orient seven of them coherently with the orientation, and one of them, say one on $S_{1}$, in the noncoherent direction (see Fig. 2). Otherwise (if some $S_{i}$ is nonorientable) orient the cycles in an arbitrary way. Next glue the surfaces together by identifying, in pairs, their boundaries as shown on Fig. 2, so that the chosen orientations on the boundaries are opposite in each of the four pairs, and so that each of the vertices on the boundary is identified with a vertex on the other surface. By performing the identifications we take care, as in Lemma 1, that at least one of the two vertices which are identified is saturated. Clearly, this is always possible since $x-1 \leq 1$, and $y-1 \leq 1$ and the four inequalities of the lemma hold.

Now it is just a matter of routine to verify that we have obtained a nonorientable surface into which some graph $G$ is quadrilaterally embedded. The graph $G$ is easily seen to be bipartite, having $m_{1}-1+m_{2}-1$ black vertices and $n_{1}-1+n_{2}-1$ white vertices. It is easily computed that precisely $k_{1}-1+y-1+k_{2}-1+x-1$ independent edges are missing up to the complete bipartite graph. This concludes the proof.

\section{Quadrilateral Embeddings}

The graph $G(m, n, k)$ can have a quadrilateral embedding into a nonorientable surface only if $m n \equiv k(\bmod 2)$. Thus we assume throughout this section that

$$
m n \equiv k \quad(\bmod 2)
$$

and, to exclude trivial and nonexisting cases, that

$$
m \geq 3, \quad n \geq 3, \quad \text { and } \quad k \leq \min (m, n) .
$$

A graph $G(m, n, k)$ is said to be admissible if the parameters $m, n, k$ satisfy conditions (2) and (3) above. Ringel [6] proved:

Lemma 3. Each admissible graph $G(m, n, 0)$ has a nonorientable quadrilateral embedding. 
Using Lemmas 1 and 3, the following lemma is easily proved.

Lemma 4. Let $G=G(m, n, 1)$ be admissible. Then $G$ has a nonorientable quadrilateral embedding if and only if $m>3$ or $n>3$.

Proof. $G(3,3,1)$ has a quadrilateral embedding into the 2 -sphere. Hence, it does not admit a quadrilateral embedding into a nonorientable surface.

For other cases assume $m \leq n$ and $n>3$. By (2), $n$ is odd, and $n \geq 5$. Therefore $G(m, n-1,0)$ is admissible, and by Lemma 3 it has a nonorientable quadrilateral embedding. The graph $G(m, 3,1)$ also has a quadrilateral embedding, for $m=3$ into the 2-sphere, and for $m>3$ into some nonorientable surface, since by Lemmas 3 and $1, N(m, 3,1)=N(m-1,3,0) \#_{2} O(3,3,1)$. Therefore, by Lemma 1 , $N(m, n, 1)=N(m, n-1,0) \#_{1} S(m, 3,1)$.

Lemma 5. Suppose that $G(m, n, k)$ is admissible, and that either $m \equiv 1(\bmod 2)$ and $k<n$, or $n \equiv 1(\bmod 2)$ and $k<m$. Then $G(m, n, k)$ has a nonorientable quadrilateral embedding in all but the following cases: $G(3,3,1), G(3,4,2)$, $G(4,3,2), G(3,5,3)$, and $G(5,3,3)$.

Proof. All the exceptional cases are planar graphs, and therefore have no quadrilateral embeddings into nonorientable surfaces.

Without loss of generality we assume that $m \equiv 1(\bmod 2)$ and $k<n$. From $(2)$ we get $n \equiv k(\bmod 2)$.

Case 1. $n=k+2$. For $m=3$ we get three exceptional cases $(k=1,2,3)$. For $m>3$ we use induction on $k$. For the base case $G(m, 3,1)$, we apply Lemma 4 . In the induction step we use Lemma 1:

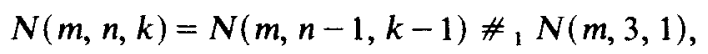

where $N(m, 3,1)$ is obtained by Lemma 4 , while for $N(m, n-1, k-1)$ the induction hypothesis applies.

Case 2. $n>k+2$. For $k=0$ we use Lemma 3, and, in general, we apply induction and Lemma 1:

$$
N(m, n, k)=N(m, n-1, k-1) \#{ }_{1} S(m, 3,1) .
$$

Lemma 6. Let $G(m, n, k)$ be admissible, and $m \equiv 0, n \equiv 0, k \equiv 0(\bmod 2)$, and suppose that $k<n$ and $k<m$. Then $G(m, n, k)$ has a nonorientable quadrilateral embedding.

Proof. We use Lemma 1 and $N(m, n, k)=N(3, n, 0) \#_{2} S(m-1, n, k)$. The first term $N(3, n, 0)$ is obtained by Lemma 3 , and $S(m-1, n, k)$ is obtained (either nonorientable, or orientable into the 2 -sphere) by Lemma 5. 
There are several remaining cases, admissible graphs $G(m, n, k)$ for which we did not prove the equality of Theorem 1 . These cases are:

(a) $G(k, k, k), k \geq 5$,

(b) $G(m, k, k), k \equiv 0(\bmod 2), m$ arbitrary, and

(c) $G(k, m, k), k \equiv 0(\bmod 2), m$ arbitrary (the dual case of (b)).

We are able to solve cases (b) and (c) with the exception of graphs $G(k+1, k, k)$ and $G(k, k+1, k)$ where $k \equiv 0(\bmod 2)$.

Lemma 7. Let $G(m, k, k)$ be admissible and $k \equiv 0(\bmod 2)$. If $m \geq k+2$ then $G(m, k, k)$ has a nonorientable quadrilateral embedding. The same holds for the dual case $G(k, m, k)$.

Proof. Induction on $m$. The basis $m=k+2$ is settled using Lemma 2. Let $m_{1}=k$, $n_{1}=k-1, k_{1}=k-2, m_{2}=4, n_{2}=3, k_{2}=2, x=y=2$. Then, the four graphs $G(k, k-1, k-2), G(4,3,2), G(k, 3,2)$, and $G(4, k-1,2)$ have quadrilateral embeddings (by Lemma 5 , or easily seen in trivial cases). The inequalities of Lemma 2 are also satisfied, thus by Lemma 2, $G(k+2, k, k)$ has a nonorientable quadrilateral embedding.

The induction step is much easier. For $m>k+2$, Lemma 1 applies:

$$
N(m, k, k)=N(m-1, k, k) \#_{2} N(3, k, 0) .
$$

Finally, we give some results for case (a), the graphs $G(k, k, k)$.

Theorem 2. For each $k>1$, the graph $G(4 k, 4 k, 4 k)$ has a nonorientable quadrilateral embedding.

Proof. Lemma 2 will be used. Take $x=y=1$ and $m_{1}=n_{1}=k_{1}=4 k-3, m_{2}=n_{2}=$ $k_{2}=5$. The graphs $G\left(m_{1}, n_{1}, k_{1}\right)$ and $G\left(m_{2}, n_{2}, k_{2}\right)$ have (orientable) quadrilateral embeddings (see [3], [5], [8]), and so have $G\left(m_{1}, n_{2}, 1\right)$ and $G\left(m_{2}, n_{1}, 1\right)$ by Lemma 4 . Thus, by Lemma 2 , the graph $G(4 k, 4 k, 4 k)$ has a nonorientable quadrilateral embedding.

The same proof as that of Theorem 2, just taking $m_{2}=n_{2}=k_{2}=7$ instead of 5 , gives us the nonorientable quadrilateral embeddings of the graphs $G(4 k+2$, $4 k+2,4 k+2), k>1$. The only problem is that we do not know if $G(7,7,7)$ has a quadrilateral embedding.

Proposition 1. If $G(7,7,7)$ has a (necessarily nonorientable) quadrilateral embedding, then all graphs $G(2 k, 2 k, 2 k), k \geq 4$, have nonorientable quadrilateral embeddings.

\section{Nonquadrilateral Embeddings}

Let $m, n \geq 3$ and $k \leq \min (m, n)$ be natural numbers such that $f(m, n, k)>0$. Suppose that $m n \neq k(\bmod 2)$, which means that the graph $G(m, n, k)$ cannot have a (nonorientable) quadrilateral embedding. 
If $k=0$ then $G(m, n, k)$ has genus $[f(m, n, k)\rceil$ as shown by Ringel [6]. Now suppose that $k>0$. We may add an edge to $G(m, n, k)$, thus obtaining the admissible graph $G(m, n, k-1)$. From $k \leq \min (m, n)$ it follows that $k-1<m$ and $k-1<n$. Thus, by the results of Section $3, G(m, n, k-1)$ has a nonorientable quadrilateral embedding. This means that $\tilde{\gamma}(G(m, n, k-1))=f(m, n, k-1)$. Now, removing the previously added edge from $G(m, n, k-1)$, we get the graph $G(m, n, k)$ embedded into the same surface. Thus

$$
\tilde{\gamma}(G(m, n, k))=f(m, n, k-1)=\lceil f(m, n, k)\rceil .
$$

This result, together with the results of Section 3, implies our Theorem 1.

\section{Nonexistence of Embeddings}

In contrast to the orientable case [5] where all nearly complete bipartite graphs have quadrilateral or nearly quadrilateral embeddings, we shall see that there are admissible graphs $G(5,4,4)$ and $G(5,5,5)$ which do not have a nonorientable equadrilateral embedding. It is interesting to note that $G(5,5,5)$ has an orientable quadrilateral embedding [5].

As far as we know, not many general methods are known, apart from the use of lengths of shortest cycles, the Euler formula, or forbidden subgraphs, for proving the nonexistence of embeddings of graphs in certain surfaces. In our opinion, investigations in this direction are a very important part of the theory of graph embeddings.

Theorem 3. The admissible graphs $G(5,4,4)$ and $G(5,5,5)$ have no nonorientable quadrilateral embedding and $\tilde{\gamma}(G(5,4,4))=f(5,4,4)+1=2$, and $\tilde{\gamma}(G(5,5,5))=$ $f(5,5,5)+1=3$.

Proof. Consider, first, $G(5,4,4)$ and assume it has a nonorientable quadrilateral embedding. Since $f(5,4,4)=1$, this embedding is into the projective plane. Let $v$ be the (black) saturated vertex of $G(5,4,4)$ and consider the neighborhood of $v$ on the surface (see Fig. 3). First we show that the black vertices $v, w, x, y, z$ are all different. Clearly, $v$ cannot match with any of the others. It is also obvious that $x \neq w$ and $x \neq y$ since we have no multiple edges. If $x=z$, this vertex would be of degree (at least) 4 , but this is impossible since $v$ is the only black vertex of $G(5,4,4)$ of degree 4 . By symmetry we conclude that $w \neq y, w \neq z$, and $y \neq z$.

In Fig. 3 all vertices of $G(5,4,4)$ are drawn but the edges (without loss of generality) $x b, y c, w a$, and $z d$ are missing. Consider any one of these edges, say $x b$. The cycle $b, y, a, x, b$ cannot bound a region, since in such a case the edge $w a$ could not be drawn on the surface. Therefore $x b$ and $w a$ (by symmetry) are embedded as shown on Fig. 3 by the dashed lines. Now, neither of the remaining two edges can be added without crossing the edges already drawn.

It is easy to construct an embedding of $G(5,4,4)$ into the Klein bottle, thus showing that $\tilde{\gamma}(G(5,4,4))=f(5,4,4)+1=2$. 


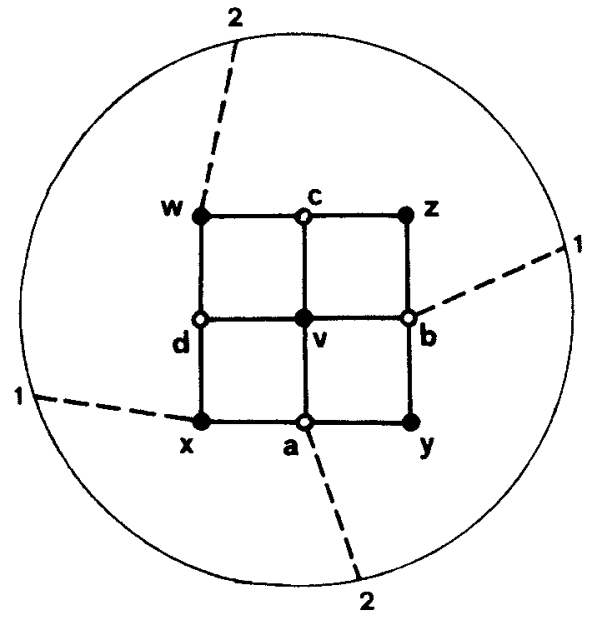

Fig. 3

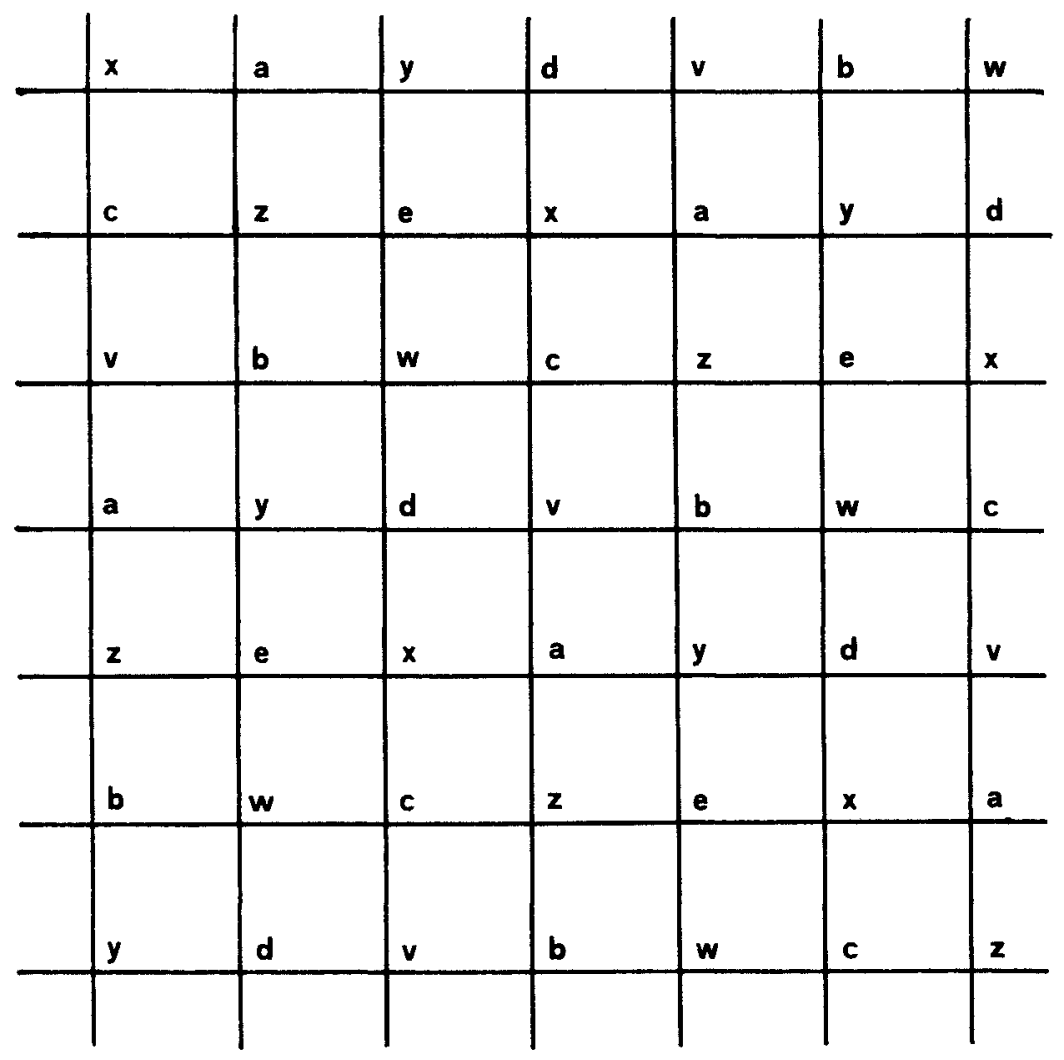

Fig. 4 
Now take $G=G(5,5,5)$. Denote the black vertices by $w, x, y, z, v$ and the white vertices by $a, b, c, d$, e in such a way that the edges $a w, b x, c y, d z$, and $e v$ are not present. Assume that $G$ has a nonorientable quadrilateral embedding (into the surface of nonorientable genus $f(5,5,5)=2$ ). Choose any black vertex, say $v$, and consider its neighborhood on the surface (see Fig. 3 and omit the dashed lines). As for $G(5,4,4)$ we can verify that the black vertices around $v$ are all different (now $x=z$ would imply that this vertex has the same four neighbors as $v$ ). This property can be used to determine, uniquely, the universal cover of the embedded graph (see Fig. 4). The universal covering factors through the double orientable cover in which each vertex $p$ of $G(5,5,5)$ has two preimages, say $p_{1}$ and $p_{2}$, such that the local rotation at $p_{1}$ is reverse to the local rotation at $p_{2}$ (when projected to the nonorientable base). Therefore the preimages of $p$ in the universal cover must be of two types, either having the local rotation as $p_{1}$ or as $p_{2}$. But from Fig. 4 it is evident that only one such rotation occurs, hence a contradiction.

Since $\gamma(G)=1$ (see [5] or Fig. 4) and $\tilde{\gamma}(G) \leq 2 \cdot \gamma(G)+1$ [8], we conclude that $\tilde{\gamma}(G(5,5,5))=3=f(5,5,5)+1$.

Remark. The above construction of the universal cover of a quadrilaterally embedded graph $G(5,5,5)$ also shows that the orientable embedding of $G(5,5,5)$ into the torus is combinatorially unique.

\section{References}

1. L. W. Beineke and R. J. Wilson, Selected Topics in Graph Theory, Academic Press, London-New York, 1978.

2. A. Bouchet, Orientable and nonorientable genus of the complete bipartite graph, $J$. Combin. Theory Ser. B 24 (1978), 24-33.

3. M. Jungerman, S. Stahl, and A. T. White, On embeddings of hypergraphs, Congr. Numer. 29 (1980), 545-557.

4. Z. Magajna, B. Mohar, and T. Pisanski, Minimal ordered triangulations of surfaces, J. Graph Theory, in press.

5. B. Mohar, T. D. Parsons, and T. Pisanski, The genus of nearly complete bipartite graphs, Ars Combin. 20B (1985), 173-183.

6. G. Ringel, Der vollständige paare Graph auf nichtorientierbaren Flächen, J. Reine Angew. Math. 220 (1965), 88-93.

7. S. Stahl, The embeddings of a graph-a survey, J. Graph Theory 2 (1978), 275-298.

8. A. T. White, Graphs, Groups and Surfaces, North-Holland, Amsterdam, 1973, 2nd edn., 1984.

F. eived May 30, 1986. 